\title{
ARTICLE
}

Clinical Study

\section{Multivariable clinical-genetic risk model for predicting venous thromboembolic events in patients with cancer}

\author{
Andrés J. Muñoz Martín ${ }^{1,2}$, Israel Ortega ${ }^{3}$, Carme Font ${ }^{2,4}$, Vanesa Pachón ${ }^{2,5}$, Victoria Castellón ${ }^{2,6}$, Virginia Martínez-Marín ${ }^{2,7}$, \\ Mercedes Salgado ${ }^{2,8}$, Eva Martínez ${ }^{2,9}$, Julia Calzas ${ }^{2,10}$, Ana Rupérez ${ }^{2,11}$, Juan C. Souto ${ }^{12}$, Miguel Martín ${ }^{1,2}$, Eduardo Salas ${ }^{3}$ and $^{2}$ \\ Jose M. Soria ${ }^{13}$
}

BACKGROUND: Venous thromboembolism (VTE) is a leading cause of death among patients with cancer. Outpatients with cancer should be periodically assessed for VTE risk, for which the Khorana score is commonly recommended. However, it has been questioned whether this tool is sufficiently accurate at identifying patients who should receive thromboprophylaxis. The present work proposes a new index, TiC-Onco risk score to be calculated at the time of diagnosis of cancer, that examines patients' clinical and genetic risk factors for thrombosis.

METHODS: We included 391 outpatients with a recent diagnosis of cancer and candidates for systemic outpatient chemotherapy. All were treated according to standard guidelines. The study population was monitored for 6 months, and VTEs were recorded. The Khorana and the TiC-Onco scores were calculated for each patient and their VTE predictive accuracy VTEs was compared.

RESULTS: We recorded 71 VTEs. The TiC-Onco risk score was significantly better at predicting VTE than the Khorana score (AUC 0.73 vs. 0.58 , sensitivity 49 vs. $22 \%$, specificity 81 vs. $82 \%$, PPV 37 vs. $22 \%$, and NPV 88 vs. $82 \%$ ).

CONCLUSIONS: TiC-Onco risk score performed significantly better than Khorana score at identifying cancer patients at high risk of VTE who would benefit from personalised thromboprophylaxis.

British Journal of Cancer (2018) 118:1056-1061; https://doi.org/10.1038/s41416-018-0027-8

\section{INTRODUCTION}

Patients with cancer (taking all types together) are at 7 times the risk of developing a venous thromboembolism (VTE); in some malignancies the risk increases to 28 times. ${ }^{1}$ The incidence of cancer-associated VTE is particularly high during the first few months after diagnosis, when distant metastases are present, and after initiating chemotherapy. ${ }^{1,2}$ VTE is a leading cause of death among patients with cancer, ${ }^{3}$ and the survival of an episode may have clinical and economic implications, including hospitalisation, potential delays in cancer therapy, recurrent VTE, post-thrombotic syndrome, and chronic thromboembolic pulmonary hypertension. Indeed, these problems are common, costly, and have a profound impact on the patient's quality of life. ${ }^{3}$

Different guidelines cover the identification of patients with cancer at risk of VTE, VTE prevention strategies, and treatment. ${ }^{4-6}$ These documents indicate that most hospitalised patients with active cancer require thromboprophylaxis throughout hospitalisation. However, in the outpatient setting, it is indicated only for high-risk patients. Outpatients with cancer should be periodically assessed for VTE risk, for which the validated risk assessment tool developed by Khorana-the Khorana score $^{7}$ is commonly recommended. However, in recent years, a number of studies have questioned whether this tool is sufficiently accurate at identifying patients who should receive thromboprophylaxis. ${ }^{8-10}$

In the present work, we hypothesised that a genetic component is involved in the appearance of VTE, a factor that the Khorana score does not take into account. Recently, the Vienna Group ${ }^{11}$ showed that the Leiden (rs6025) variant of the gene coding for factor $V$ in the coagulation pathway doubles the risk of a VTE event occurring in patients with cancer. This variant might, therefore, provide a promising biomarker of venous thrombosis in such patients, and could be used in individual risk prediction. ${ }^{12}$ Other genes are known to increase the risk of VTE in the general population, and a tool (the Thrombo inCode or TiC tool) involving them as markers has been developed to predict VTE-but only for

\footnotetext{
${ }^{1}$ Medical Oncology Service, Hospital General Universitario Gregorio Marañón, C/ Doctor Esquerdo, 46, 28007 Madrid, Spain; ${ }^{2}$ Cancer \& Thrombosis Working Group, Spanish

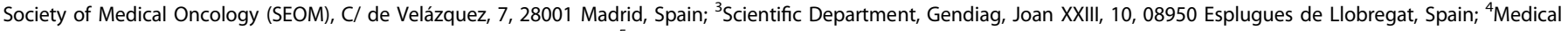

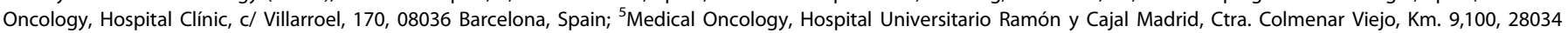

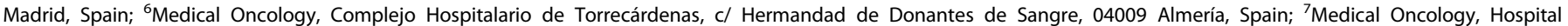

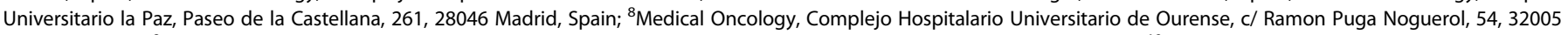

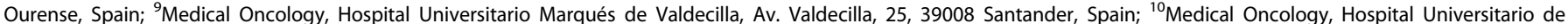

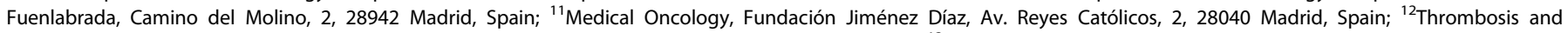

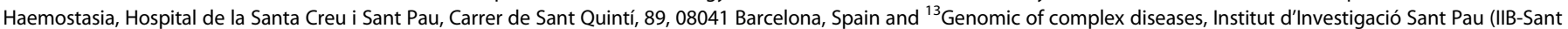
Pau), Carrer de Sant Quintí, 89, 08041 Barcelona, Spain

Correspondence: Andrés J. Muñoz Martín (andresmunmar@hotmail.com)
}

Received: 25 September 2017 Revised: 17 January 2018 Accepted: 22 January 2018

Published online: 28 March 2018 
Table 1. Study population characteristics

\begin{tabular}{|c|c|c|c|}
\hline & VTE & No-VTE & $p$-value \\
\hline N & 71 & 320 & \\
\hline Sex (female), $n(\%)$ & $27(38.0)$ & $108(33.8)$ & 0.584 \\
\hline Age, mean (sd) & $64.1(11.0)$ & $64.3(10.5)$ & 0.903 \\
\hline Diabetes, $n(\%)$ & $12(16.9)$ & $62(19.4)$ & 0.754 \\
\hline Smoking, $n(\%)$ & $21(29.6)$ & $66(20.6)$ & 0.138 \\
\hline Family history (\%) & $6(8.4)$ & $12(3.7)$ & 0.112 \\
\hline $\mathrm{BMI}>25, n(\%)$ & $36(50.7)$ & $144(45.0)$ & 0.459 \\
\hline Hypercholesterolemia, $n$ (\%) & $29(40.8)$ & $106(33.1)$ & 0.271 \\
\hline Hypertension (\%) & $33(46.5)$ & $141(44.1)$ & 0.932 \\
\hline Khorana $\geq 3$ & $16(22.5)$ & $58(18.1)$ & 0.505 \\
\hline \multicolumn{4}{|l|}{ Primary site of tumour: } \\
\hline Colon & $22(31.0)$ & $141(44.1)$ & 0.059 \\
\hline Pancreas & $29(40.8)$ & $43(13.4)$ & $<0.001$ \\
\hline Lung & $11(15.5)$ & $76(23.8)$ & 0.175 \\
\hline Oesophagus & $2(2.8)$ & $12(3.7)$ & 0.976 \\
\hline Stomach & $7(9.9)$ & $48(15.0)$ & 0.348 \\
\hline \multicolumn{4}{|l|}{ Tumour Stage: } \\
\hline $\mathrm{I}+\mathrm{II}$ & $5(7.0)$ & $66(20.6)$ & 0.012 \\
\hline III & $18(25.4)$ & $121(37.8)$ & 0.065 \\
\hline IV & $48(67.6)$ & $133(41.6)$ & $<0.001$ \\
\hline Haemoglobin <100 g/L, n (\%) & $4(5.6)$ & $18(5.6)$ & $>0.999$ \\
\hline Platelet $>350 \times 10^{9} / \mathrm{L}, \mathrm{n}(\%)$ & $13(18.3)$ & $74(23.1)$ & 0.469 \\
\hline Leukocyte $>11 \times 10^{9} / \mathrm{L}$ & $15(21.1)$ & $58(18.1)$ & 0.675 \\
\hline \multicolumn{4}{|l|}{ SNPs, risk alleles (\%) } \\
\hline \multicolumn{4}{|l|}{ F5 rs6025 } \\
\hline 0 Risk Alleles & $68(95.8)$ & $314(98.1)$ & 0.213 \\
\hline 1 Risk Allele & $3(4.2)$ & $6(1.9)$ & \\
\hline \multicolumn{4}{|l|}{ F5 rs4524 } \\
\hline 0 Risk Alleles & $1(1.4)$ & $22(6.9)$ & 0.108 \\
\hline 1 Risk Allele & $22(31.0)$ & $115(35.9)$ & \\
\hline 2 Risk Alleles & $48(67.6)$ & $183(57.2)$ & \\
\hline \multicolumn{4}{|l|}{ F2 rs1799963 } \\
\hline 0 Risk Alleles & $69(97.2)$ & 307 (95.9) & $>0.999$ \\
\hline 1 Risk Allele & $2(2.8)$ & $12(3.7)$ & \\
\hline 2 Risk Alleles & 0 & $1(0.3)$ & \\
\hline \multicolumn{4}{|l|}{ F12 rs1801020 } \\
\hline 0 Risk Alleles & $46(64.8)$ & $204(63.7)$ & $>0.999$ \\
\hline 1 Risk Allele & $23(32.4)$ & $103(32.2)$ & \\
\hline 2 Risk Alleles & $2(2.8)$ & $13(4.1)$ & \\
\hline \multicolumn{4}{|l|}{ F13 rs5985 } \\
\hline 0 Risk Alleles & $36(50.7)$ & $184(57.5)$ & 0.514 \\
\hline 1 Risk Allele & $30(42.3)$ & $119(37.2)$ & \\
\hline 2 Risk Alleles & $5(7.0)$ & $17(5.3)$ & \\
\hline \multicolumn{4}{|l|}{ SERPINC1 rs121909548 } \\
\hline 0 Risk Alleles & $71(100.0)$ & 319 (99.7) & $>0.999$ \\
\hline 1 Risk Allele & 0 & $1(0.3)$ & \\
\hline \multicolumn{4}{|l|}{ SERPINA10 rs2232698 } \\
\hline 0 Risk Alleles & $68(95.8)$ & $314(98.1)$ & 0.213 \\
\hline 1 Risk Allele & $3(4.2)$ & $6(1.9)$ & \\
\hline \multicolumn{4}{|l|}{ A1 blood group } \\
\hline o A1 Allele & $41(57.7)$ & $194(60.6)$ & 0.776 \\
\hline $1 \mathrm{~A} 1$ Allele & $24(33.8)$ & $105(32.8)$ & \\
\hline 2 A1 Alleles & $6(8.4)$ & $21(6.6)$ & \\
\hline
\end{tabular}

non-oncological populations. ${ }^{13}$ The use of such tools ought to allow more tailored thromboprophylaxis strategies to be followed.

The present work proposes a TiC-derived risk score-the TiCOnco risk score-which takes into account both genetic and clinical risk factors, and which can be used to identify patients with cancer in the outpatient setting who are at high risk of VTE. Its capacity to identify such patient was compared with that of the Khorana score. The results suggest that the TiC-Onco risk score can better predict which patients should receive thromboprophylaxis.

\section{PATIENTS AND METHODS}

Study design and participants

The study protocol was approved by the participant hospitals' institutional review boards. Signed informed consent was obtained from each patient.

This study-the ONCOTHROMB12-01 study (Clinicaltrial.gov indentifier: NCT03114618)-is an observational cohort study involving an 18 month monitoring period with analysis at 6, 12, and 18 months. This paper presents the results for the first 6 months.

Selection criteria were as follows:

- Over 18 years of age

- Recent diagnosis of cancer of the following types: colorectal, oesophago-gastric, lung, or pancreatic.

- ECOG/WHO/Zubrod score of 0-2

- Candidates for systemic outpatient chemotherapy according to standard guidelines.

- No outpatient thromboprophylactic therapy deemed mandatory by the treating oncologist.

The Khorana score (reference tool) and the proposed TiC-Onco score (index tool) were calculated for each patient at the moment of initial diagnosis and their accuracy in terms of predicting the observed VTE events of the two tools was compared.

Diagnosis of VTE events

Deep vein thrombosis in the lower limbs was diagnosed by ultrasound or ascending venography. Pulmonary embolism was diagnosed by ventilation-perfusion lung scanning, pulmonary angiography, or spiral computed tomography. Intracranial venous thrombosis was diagnosed by magnetic resonance imaging.

Development of the TiC-Onco risk score

The TiC-Onco risk score tool was developed in three steps:

1. Development of a genetic risk score.

A total of 391 patients were genotyped for the genes shown in Table 1 using blood extracted at the time of diagnosis, employing TaqMan genotyping assays and the EP1 Fluidigm platform (an efficient endpoint PCR system for high-sample-throughput SNP genotyping). At 6 months, multivariate logistic regression analysis was performed to determine the weight of each genetic variable in the appearance of a VTE event. The final genetic risk score was determined using the genetic variants associated with an increased risk of VTE in the multivariate model $(p \leq 0.25)$.

2. Selection of clinical variables associated with the development of VTE.

Data were collected from all patients on the clinical risk factors cited in the literature ${ }^{14}$ as being associated with VTE and that could be known at the time of diagnosis: primary tumour site, tumour node metastasis stage, and body mass index (BMI), use of tobacco, age, sex, family (first degree) history of VTE, the presence of diabetes, hypertension, and high blood cholesterol level, the Khorana score, previous surgery, number of platelets, number of leukocytes, and immobilisation. The risk of VTE associated with the 
Table 2. Population charactheristics per cancer type

\begin{tabular}{|c|c|c|c|c|c|c|}
\hline Tumour type & Colon & Pancreas & Lung & Oesophagus & Stomach & Total \\
\hline Patients (\%) & 41.69 & 18.41 & 22.25 & 3.58 & 14.07 & 100 \\
\hline VTE & 22 & 29 & 11 & 2 & 7 & 71 \\
\hline$\%$ VTE & 13.50 & 40.28 & 12.64 & 14.29 & 12.73 & 18.16 \\
\hline Stage I + II & 29 & 18 & 9 & 3 & 12 & 71 \\
\hline Stage IV & 60 & 42 & 44 & 6 & 29 & 181 \\
\hline Death, $n(\%)$ & $13(7.98)$ & $24(33.33)$ & $13(14.94)$ & $1(7.14)$ & $7(12.73)$ & \\
\hline VTE death, $n(\%)$ & $5(38.46)$ & $14(58.33)$ & $3(23.08)$ & 0 & 0 & \\
\hline No-VTE deaths, $n(\%)$ & $8(61.54)$ & $10(41.67)$ & $10(76.92)$ & $1(100)$ & $7(100)$ & \\
\hline
\end{tabular}

primary tumour site (low, high, and very high) was categorised as when determining the Khorana score. ${ }^{15}$ The risks associated with platelet and leukocyte numbers were categorised using the same cut-offs as for the Khorana score. ${ }^{15}$

At 6 months, univariate analysis was performed to determine which of these variables were associated with the appearance of a VTE event. Those associated with an increased risk of VTE ( $p \leq$ $0.25)$ were selected.

3. Development of the clinical-genetic model.

The genetic risk score and the clinical variables selected were subjected to multivariate logistic regression analysis using an AICbased backward selection process. ${ }^{16}$

Internal validation

Internal validation to obtain the degree of optimism in the area under the receiver operating characteristic (ROC) curve (AUC) estimation was done using the bootstrap approach, ${ }^{17}$ considering 100 resamples from the original data.

Comparing the Khorana and TiC-Onco risk scores

The risk prediction capacity of the Khorana and TiC-Onco risk scores was evaluated using the area under the receiver operating characteristic (ROC) curve (AUC, larger values indicate better discrimination). ${ }^{18}$ Standard measures of sensitivity, specificity, positive, and negative predictive value (PPV and NPV), and positive and negative likelihood ratios (PLR and NLR), ${ }^{19}$ were determined for specific cut-off points.

For Khorana score the cut-off defining high risk was set at $\geq 3$ (the normal cut-off value), and 0 for the low risk category definition. We contemplate two scenarios when determining a cut-off for the TiC-Onco score. In the first one (the main scenario), the cut-off is selected as the point on the ROC curve giving the same specificity as provided by the Khorana score (around 80\%). It defines those individuals who are in high risk. This will be the default cut-off for TiC-Onco when not specified in the text. In this scenario, we also determine a second cut-off to classify the nonhigh risk individuals into either intermediate or low risk. Thus, we allow the TiC-Onco score to provide three risk categories-high, intermediate, and low risk. This second cut-off to discriminate between low and intermediate categories is selected as the point giving a sensitivity of $90 \%$. The second scenario is presented just for informative purposes. In this case, the cut-off is selected as the point which maximises the Youden's index (defined as sensitivity + specificity-1). ${ }^{20}$ In this scenario, we only consider a TiC-Onco with two categories, high risk and non-high risk.

\begin{tabular}{|lr|}
\hline Table 3. Clinical-genetic risk score Thrombo inCode-Oncology (TiC- \\
Onco) & -value \\
\hline Variable & 0.0049 \\
\hline GRS & 0.0658 \\
BMI >25 & 0.1076 \\
Family history & \\
Primary tumour site & 0.3483 \\
$\quad$ HR & 0.0033 \\
VHR & 0.0003 \\
Tumour & \\
stage & \\
GRS & 0.1460 \\
$\quad$ rs2232698 & 0.2064 \\
rs6025 & 0.2003 \\
rs5985 & 0.0396 \\
\hline rs4524 & \\
\hline GRS Genetic Risk Score, HR High Risk, VHR Very High Risk \\
\hline
\end{tabular}

Statistical analysis

Continuous variables were recorded as median [1st-3rd Quartiles], and categorical variables as proportions. Univariate association between clinical/genetic variables and events was determined using either $t$-test or Wilcoxon rank sum tests for continuous variables, and $x^{2}$ or Fisher tests for categorical variables. All calculations were performed using $\mathrm{R}$ statistical software (version 3.1.3). ${ }^{21}$

Number of patients needed to treat

To assess the effect of the TiC-Onco risk score in terms of preventing VTE events, the number of patients needed to treat (NNT) was determined for both scores. ${ }^{22}$ It was assumed that prophylactic medication would reduce cancer-associated VTE by $46 \%{ }^{23}$

\section{RESULTS}

Patient characteristics

Tables 1 and 2 show the clinical and demographic characteristics of the 391 patients at the start of the study. For each variable, the 
Table 4. Predictive capability of TiC-Onco and Khorana scores

\begin{tabular}{llllll}
\hline & TiC-Onco (1) & TiC-Onco (2) & Khorana & $p$ (TiC-Onco (1) vs Khorana) & $p$ (TiC-Onco (2) vs Khorana) \\
\hline AUC $(95 \%$ Cl) & $0.734(0.67-0.79)$ & $0.734(0.67-0.79)$ & $0.580(0.51-0.65)$ & $<0.001$ & $<0.001$ \\
Sensitivity, \% (95\% Cl) & $49.30(37.7-60.9)$ & $85.92(77.8-94.0)$ & $22.54(12.8-32-3)$ & $<0.001$ & $<0.001$ \\
Specificity, \% (95\%, Cl) & $81.25(77.0-85.5)$ & $49.06(43.6-54.5)$ & $81.76(77.5-86.0)$ & 0.823 & $<0.001$ \\
PPV, \% (95\% Cl) & $36.84(27.1-46.5)$ & $27.23(21.4-33.1)$ & $21.62(12.2-31.0)$ & 0.004 & 0.218 \\
NPV, \% (95\% Cl) & $87.84(84.1-91.6)$ & $94.01(90.4-97.6)$ & $82.54(78.3-86.7)$ & $<0.001$ & $<0.001$ \\
PLR (95\% Cl) & $2.63(1.89-3.65)$ & $1.69(1.46-1.95)$ & $1.24(0.76-2.02)$ & 0.005 & 0.244 \\
NLR (95\% Cl) & $0.62(0.49-0.79)$ & $0.29(0.16-0.52)$ & $0.95(0.83-1.09)$ & 0.001 & $<0.001$ \\
\hline
\end{tabular}

TiC-Onco (1) shows the predictive capabilities for the default cut-off (see Methods). TiC-Onco (2) shows the predictive capabilities for the cut-off providing the best Youden's Index. AUC Area Under the Roc Curve, PPV Positive Predictive Value, NPV Negative Predictive Value, PLR Positive Likelihood Ratio, NLR Negative Likelihood Ratio

\begin{tabular}{lllllll|}
\hline $\begin{array}{l}\text { Table 5. } \\
\text { patients }\end{array}$ & \begin{tabular}{l} 
Patient distribution according to Khorana score considering \\
\hline Khorana
\end{tabular} & VTE & No-VTE & Patients ( $n$ ) & Patients (\%) & $\%$ VTE \\
\hline 0 & 14 & 94 & 108 & 27.62 & 12.96 \\
1 & 11 & 74 & 85 & 21.74 & 12.94 \\
2 & 30 & 92 & 122 & 31.20 & 24.59 \\
$\geq 3$ & 16 & 58 & 74 & 18.93 & 21.62 \\
NA & 0 & 2 & 2 & 0.51 & 0 \\
Total & 71 & 320 & 391 & & \\
\hline $\begin{array}{l}\text { Patients (\%) percentage of patients per Khorana score level. \%VTE cases } \\
\text { percentage of patients with VTE in relation to the number of patients per } \\
\text { Khorana score level }\end{array}$
\end{tabular}

number and percentage of patients who experienced a VTE, or not, at some point in the 6-month study period, are shown. The overall incidence of VTE was $18 \%$. Patients suffering from pancreatic cancer experienced VTE at a significantly higher frequency $(40 \%)$ than patients with other type of cancers $(p<$ 0.001) (Table 2).

Development of the TiC-Onco risk model

Table 3 shows the genetic and clinical markers that were significantly associated by multivariate analysis with a VTE event, and thus selected for inclusion in the TiC-Onco risk score model.

Accuracy and validation of the risk model

The TiC-Onco score showed an AUC of $0.73(0.67-0.79)$, a sensitivity of $49 \%$, and a specificity of $81 \%$. Its PPV was $37 \%$, NPV 88\%, PLR 2.6, and NLR 0.6\% (Table 4). The Khorana score showed a significantly lower capacity to distinguish between patients who experienced/did not experience a VTE event (AUC 0.73 vs. $0.58 ; p<0.001$ ). The sensitivity of the TiC-Onco score was significantly higher than that of the Khorana (49 vs. $22 \%$; $p<$ 0.001 ), while the specificities of both scores were similar (81 vs. $82 \% ; p=0.823$ ). The PPV and NPV of the TiC-Onco score were significantly higher than those of the Khorana score (37 vs. $22 \%$; $p$ $=0.004$ for PPV and 88 vs. $82 \% ; p<0.001$ for NPV). The LRs of the TiC-Onco score were also significantly better (Table 4).

Table 5 shows the distribution of patients with or without VTE according to the Khorana score. The great majority of patients who suffered a VTE event (77\%) were identified by the Khorana score as being at low or moderate risk (values 0,1 , and 2). Among these 55 patients, however, 17 (31\%) were detected as high-risk patients by the TiC-Onco score. When the cut-off for high risk was taken as the best Youden Index, the TiC-Onco score returned significantly better predictions of risk than the Khorana score, especially in terms of sensitivity ( $86 \mathrm{vs.} 22 \%, p<0.001$ ) (Table 4). In this scenario, of the 55 patients who experienced a VTE event (but who were classified as not being at high-risk by the Khorana score), $40(73 \%)$ were detected as high risk patients by the TiCOnco score. Table 6 shows rates of VTE according to prespecified risk categories for both $\mathrm{TiC}-$ Onco and Khorana.

The NNT values for: (a) if all patients included in the study had been treated (NNT = 12); (b) if only the patients with a Khorana score of $\geq 3$ had been treated (NNT $=10$ ), or (c) if only patients with a high risk TiC-Onco score (with the cut-off set at the same specificity as the Khorana score) had been treated (NNT $=6)$.

\section{DISCUSSION}

When deciding whether to use primary antithrombotic prophylaxis in outpatients with cancer who are candidates for chemotherapy, a clinician needs to determine the risk of VTE and weigh the likely benefit against the risk of bleeding. Despite the awareness of scientific societies regarding cancer-associated VTE, thromboprophylaxis is limited among outpatients, probably due to the sub-optimal predictive capacity of the existing tools used to predict the risk of experiencing a VTE event. The present work presents a new predictive score, the TiC-Onco score, which shows significantly better predictive power in this regard than the Khorana score.

The incidence of VTE in the present population at 6 months of follow-up was $18 \%$ (occurring in $71 / 391$ patients); this is within the range of figures cited in a previous publication ${ }^{24}$ for the same follow-up period. It is also in agreement with the observation that the incidence of VTE is highest among patients with pancreatic cancer. ${ }^{25}$ However, no other clear differences between tumour types were seen with respect to the Khorana score, probably because a large proportion (46\%) of the present patients had stage IV tumours.

The statistical analysis of the present data detected four genetic variants that were independently associated with VTE in outpatients with cancer (Table 2). These were combined into the algorithm for the Tic-Onco score, which initially allowed the patients to be classified as either at high or low risk of VTE. Among the patients in the TiC-Onco high risk group, 37\% eventually suffered a VTE event, while $12 \%$ of those in the low risk group experienced the same (Table 4). However, when the three-tier TicOnco risk category system was contemplated (explained in Methods), $37 \%$ of the high risk, $18 \%$ of the moderate risk, and $6 \%$ of the low risk patients experienced a VTE event. In comparison, 22,20 , and $13 \%$ of the patients in the equivalent 
Table 6. Percentage of study population among risk categories, and percentage of patients with VTE. See Methods for details about the definition of two and three categories

\begin{tabular}{llllrr}
\hline & \multicolumn{2}{l}{ TiC-Onco } & & \multicolumn{2}{l}{ Khorana } \\
\cline { 2 - 3 } \cline { 5 - 6 } & \% of cancer population & \% of cancer patient with VTE & & \% of cancer population & \% of cancer patient with VTE \\
\hline High risk & 24.30 & 36.84 & 12.16 & 18.93 & 21.62 \\
Non-high risk & 75.70 & 18.30 & 52.94 & - \\
Moderate risk & 39.13 & 5.59 & 27.62 & 19.81 \\
Low risk & 36.57 & & &
\end{tabular}

Khorana score categories experienced an event. The result obtained for the high risk Khorana score $\geq 3(22 \%)$ is similar to that reported for high risk group at 6 months by other authors $(18 \%)(p=0.6))^{26,27}$

The majority of genetic studies have excluded individuals with cancer-related thrombosis, and the relatively few studies that have been performed (which have mainly focused on the factor $V$ Leiden and prothrombin G20210A genetic variants), have reported conflicting results. ${ }^{1,11,12,28-30}$ These discrepancies are most likely due to the use of a single-marker, and inherent problems of low statistical power and poor reproducibility. The present work overcomes these problems by using several markers based on previous knowledge, a strategy that provided good results in previous work performed with non-oncology patients. ${ }^{13}$

Although the presently noted distribution of patients in the different Khorana risk categories is similar to that previously reported for the same follow-up time by other authors, ${ }^{26,} 27$ the predictive power of the TiC-Onco was found to be significantly greater than that of the Khorana Score. This superiority is demonstrated by a better AUC, better likelihood ratios, a higher PPV and NPV, and, importantly, a much higher sensitivity (49\%).

In summary, this paper reports a clinical-genetic risk score that is significantly better than the Khorana score at identifying outpatients with cancer at high risk for experiencing a VTE event. Patients identified as being at high risk by the Tic-Onco risk score (using a specificity equal to that provided by the Khorana index as the cut-off) would likely benefit from thromboprophylaxis despite the risk of haemorrhage; they should, therefore, be seen as candidates for prophylactic treatment for VTE. The lower NNT of the Tic-Onco score reveals it can identify those patients most likely to benefit from prophylaxis. It is important that an accurate predictive tool like the TiC-Onco score be available if the morbidity associated with VTE is to be reduced, and because patients with cancer who experience a VTE are more likely to die than those who do not (31 vs. $11 \%$ in the present study; $p<0.001$ ).

The reduced number of cancer types included might be seen as a weakness of the present study. However, it has the strengths of a multi-site setting, and a large proportion of patients in the advanced stages of tumour node metastasis classification.

As the risk of cancer-associated VTE is high even 6 months before cancer diagnosis, and the peak incidence is from 0 to 6 months $^{1,31}$ post-diagnosis, it is recommended that the TiC-Onco score be calculated at the moment cancer is suspected. ${ }^{32,33}$

\section{ACKNOWLEDGEMENTS}

The authors thank the Sociedad Española de Oncología Médica, the European Society of Cardiology, the Fundación Josep Carreras contra la Leucemia, the Sociedad Española de Trombosis y Hemostasia, CERC Programme/Generalitat, Ferrer inCode, and Leo-Pharma for funding and institutional support. The authors thank Adrian Burton (www.physicalevidence.es) for editorial assistance. The study has been presented in part in: ISTH congress (Toronto-2015), ICTHIC congress (Bergamo-2016), SEOM congress (Madrid-2016), WHITH congress (Barcelona-2017), and ISTH (Berlin2017).

\section{ADDITIONAL INFORMATION}

Competing interests: A.M., J.M.S., and E.S. are inventors in a patent application claiming the use of TiC-Onco. A.M. and Jose M.S will receive royalties for giving the right to Gendiag of being the assignee of that patent application. Israel Ortega and Eduardo Salas are employees of Gendiag. C.F., V.P., V.C., V.M-M., M.S., E.M., J.C., A.R., M. M., and J.C.S. have no conflic of interest.

Funding: This work was supported by Leo Pharma; Ferrer inCode; Fundación Josep Carreras; European Society of Cardiology; and the Spanish Society of Thrombosis and Haemostasia.

\section{REFERENCES}

1. Blom, J. W., Doggen, C. J. M., Osanto, S. \& Rosendaal, F. R. Malignancies, prothrombotic mutations, and the risk of venous thrombosis. JAMA 293, 715-722, (2005).

2. Khorana, A. A., Dalal, M., Lin, J. \& Connolly, G. C. Incidence and predictors of venous thromboembolism (VTE) among ambulatory high-risk cancer patients undergoing chemotherapy in the United States. Cancer 119, 648-655, (2013).

3. Noble, S. \& Pasi, J. Epidemiology and pathophysiology of cancer-associated thrombosis. Br. J. Cancer 102, S2-S9, (2010).

4. Lyman, G. H. et al. Venous thromboembolism prophylaxis and treatment in patients with cancer: American Society Of Clinical Oncology Clinical Practice Guideline Update 2014. J. Clin. Oncol. 33, 654-656, (2015).

5. Muñoz Martín, A. J., Font Puig, C., Navarro Martín, L. M., Borrega García, P. \& Martín Jiménez, M. Clinical guide SEOM on venous thromboembolism in cancer patients. Clinical Transl. Oncol. 16, 1079-1090, (2014).

6. Falanga, A., Roila, F. \& Mandala, M. Management of venous thromboembolism (VTE) in cancer patients: ESMO Clinical Practice Guidelines clinical practice guidelines. Ann. Oncol. 22 22, 85-92, (2011).

7. Khorana, A. A., Kuderer, N. M., Culakova, E., Lyman, G. H. \& Francis, C. W. Development and validation of a predictive model for chemotherapy-associated thrombosis.Blood 111, 4902-4908 (2015).

8. Muñoz Martín, A. J. et al. Incidence of venous thromboembolism (VTE) in ambulatory pancreatic cancer patients receiving chemotherapy and analysis of Khorana's predictive model. Clinical \& Transl. Oncol.16, 927-930, (2014).

9. Srikanthan, A. et al. Large retroperitoneal lymphadenopathy as a predictor of venous thromboembolism in patients with disseminated germ cell tumors treated with chemotherapy. Journal. clinical Oncol. 33, 582-587, (2015).

10. Ugarte Fornell, G. et al. Predictive Khorana's model in patients with venous thromboembolic disease and cancer. Med. clínica 141, 479-481, (2013).

11. Pabinger, I. et al. Factor $V$ Leiden mutation increases the risk for venous thromboembolism in cancer patients-results from the Vienna Cancer And Thrombosis Study (CATS). Journal. Thromb. Haemost. 13, 17-22, (2015).

12. Tinholt, M., Sandset, P. M. and Iversen, N. (2016) Polymorphisms of the coagulation system and risk of cancer, Thromb. Res. S49-S54. https://doi.org/10.1016/ S0049-3848(16)30098-6.

13. Soria, J. M. et al. Multilocus genetic risk scores for venous thromboembolism risk assessment. Journal. Am. Heart Assoc. 3, e001060, (2014).

14. Falanga, A. \& Russo, L. Epidemiology, risk and outcomes of venous thromboembolism in cancer. Hamostaseologie 32, 115-125, (2012).

15. Khorana, A. A., Kuderer, N. M., Culakova, E., Lyman, G. H. \& Francis, C. W. Development and validation of a predictive model for chemotherapy-associated thrombosis. Blood 111, 4902-4907, (2008).

16. Venables, W. N. and Ripley, B. D. (2002) Modern Applied Statistics with S: Springer New York (Statistics and Computing). https://doi.org/10.1007/978-0-387-21706-2.

17. Smith, G. C., Seaman, S. R., Wood, A. M., Royston, P. \& White, I. R. Correcting for optimistic prediction in small data sets. Am. J. Epidemiol. 180, 318-324, (2014). 
18. Hanley, Ja \& Hajian-Tilaki, K. O. Sampling variability of nonparametric estimates of the areas under receiver operating characteristic curves: an update. Acad. Radiol. 4, 49-58, (1997).

19. Attia, J. Moving beyond sensitivity and specificity: using likelihood ratios to. Aust. Prescr. 26, 111-113 (2003).

20. Schisterman, E. F., Perkins, N. J., Liu, A. \& Bondell, H. Optimal cut-point and its corresponding Youden Index to discriminate individuals using pooled blood samples. Epidemiology 16, 73-81 (2005).

21. R Development Core Team (2015) R: a Language and Enviroment for Statistical Computing. (R Foundation for Statistical Computing, Vienna, 2015).

22. Sackett, D. L. \& Haynes, R. B. Summarizing the effects of therapy: a new table and some more terms. Acp. J. Club. 127, A15-A16 (1997).

23. Di Nisio, M., Porreca, E., Otten, H.-M. \& Rutjes, A. W. S. Primary prophylaxis for venous thromboembolism in ambulatory cancer patients receiving chemotherapy. Cochrane Database Syst. Rev. 8, CD008500, (2014).

24. Streiff, M. B. et al Cancer-associated venous thromboembolic disease, version 1.2015. Journal. Natl. Compr. Cancer Netw. 13, 1079-1095 (2015).

25. Qureshi, W. et al. Venous thromboembolism in cancer: an update of treatment and prevention in the era of newer anticoagulants. Front. Cardiovasc. Med. Front. 3, 24, (2016).

26. Ay, C. et al. Prediction of venous thromboembolism in cancer patients. Blood 116 , 5377-5382, (2010).

27. Thaler, J., Ay, C. \& Pabinger, I. Venous thromboembolism in cancer patients- Risk scores and recent randomised controlled trials. Thromb. Haemost. 108 1042-1048, (2012).

28. Pihusch, R. et al. Impact of thrombophilic gene mutations on thrombosis risk in patients with gastrointestinal carcinoma. Cancer 94, 3120-3126, (2002).

29. Gran, O. V. et al. Joint effects of cancer and variants in the factor 5 gene on the risk of venous thromboembolism. Haematologica 101, 1046-1053, (2016).
30. Kennedy, M. et al. Factor V Leiden, prothrombin $20210 \mathrm{~A}$ and the risk of venous thrombosis among cancer patients. Br. J. Haematol. 128, 386-388, (2005).

31. Khorana, A. A., Dalal, M., Lin, J. \& Connolly, G. C. Incidence and predictors of venous thromboembolism (VTE) among ambulatory high-risk cancer patients undergoing chemotherapy in the United States. Cancer 119, 648-655, (2013b).

32. Gran, O. V. et al. Joint effects of cancer and variants in the factor 5 gene on the risk of venous thromboembolism. Haematol. Haematol. 101, 1046-1053, (2016).

33. Lyman, G. H., Bohlke, K. \& Falanga, A., American Society of Clinical Oncology. Venous thromboembolism prophylaxis and treatment in patients with cancer: American Society of Clinical Oncology clinical practice guideline update. Journal. Oncol. Pract. 11, e442-e444, (2015). Attribution 4.0 International License, which permits use, sharing, adaptation, distribution and reproduction in any medium or format, as long as you give appropriate credit to the original author(s) and the source, provide a link to the Creative Commons license, and indicate if changes were made. The images or other third party material in this article are included in the article's Creative Commons license, unless indicated otherwise in a credit line to the material. If material is not included in the article's Creative Commons license and your intended use is not permitted by statutory regulation or exceeds the permitted use, you will need to obtain permission directly from the copyright holder. To view a copy of this license, visit http://creativecommons. org/licenses/by/4.0/.

(c) The Author(s) 2018

(1061 